\title{
Directed subgraph complexes
}

\author{
Axel Hultman* \\ Fachbereich Mathematik und Informatik \\ Philipps-Universität Marburg \\ D-35032 Marburg, Germany \\ axel@mathematik.uni-marburg.de
}

Submitted: Nov 25, 2003; Accepted: Oct 18, 2004; Published: Oct 26, 2004

Mathematics Subject Classifications: 05C20, 05C40, 55P15

\begin{abstract}
Let $G$ be a directed graph, and let $\Delta_{G}^{A C Y}$ be the simplicial complex whose simplices are the edge sets of acyclic subgraphs of $G$. Similarly, we define $\Delta_{G}^{N S C}$ to be the simplicial complex with the edge sets of not strongly connected subgraphs of $G$ as simplices. We show that $\Delta_{G}^{A C Y}$ is homotopy equivalent to the $(n-1-k)$-dimensional sphere if $G$ is a disjoint union of $k$ strongly connected graphs. Otherwise, it is contractible. If $G$ belongs to a certain class of graphs, the homotopy type of $\Delta_{G}^{N S C}$ is shown to be a wedge of $(2 n-4)$-dimensional spheres. The number of spheres can easily be read off the chromatic polynomial of a certain associated undirected graph.

We also consider some consequences related to finite topologies and hyperplane arrangements.
\end{abstract}

\section{Introduction}

A monotone property of a (directed or undirected) graph is one which is preserved under deletion of edges. Hence, the set of all graphs on a particular vertex set, $[n]$ say, that satisfy a monotone property form a simplicial complex whose vertex set is the set of edges of the graphs. In numerous recent papers, see e.g. $[1,5,6,10,11,14,15]$, the topological properties of such complexes of graphs have been studied. Although most papers have dealt with complexes of all graphs having a particular property $P$, it is indeed natural to study the complex of all subgraphs of a given graph that satisfy $P$. The purpose of this paper is to study directed graph complexes of this type. The properties that we

*Partially supported by the European Commission's IHRP Programme, grant HPRN-CT-2001-00272, "Algebraic Combinatorics in Europe". 
focus on are acyclicity and strong non-connectivity. Both were studied by Björner and Welker [5] in the case of all graphs. We adapt their techniques in order to generalize their results. We also consider some consequences related to topics such as finite topologies and hyperplane arrangements.

In Section 3 we study acyclic graphs. The homotopy type of the complex of acyclic subgraphs of any given directed graph is determined. It is either a homotopy sphere or contractible. Thereafter, in Section 4, we focus on not strongly connected graphs. More precisely, we compute the homotopy type of the complex of not strongly connected subgraphs of a directed graph, if the graph belongs to a particular class, which we call 2-dense graphs.

We begin, however, with a brief survey in the next section of the more or less standard tools from topological combinatorics that will be made use of later.

Acknowledgement. The author is grateful to his advisor Anders Björner for suggesting the study of subgraph complexes.

\section{Basic topological combinatorics}

Here, we briefly review the parts of the topological combinatorics machinery that we will use later. For more details we refer to the survey [4].

To any poset $P$, we associate the order complex $\Delta(P)$. It is the simplicial complex whose faces are the chains of $P$. Similarly, to any simplicial complex $\Sigma$, we associate its face poset $P(\Sigma)$ which consists of the nonempty faces of $\Sigma$ ordered by inclusion. The complex $\Delta(P(\Sigma))$ is the barycentric subdivision of $\Sigma$, hence it is homeomorphic to $\Sigma$. (We do not distinguish notationally between a complex and its underlying topological space.)

Our first two tools are due to Quillen [12]. In a poset $P$ with an element $x \in P$, we write $P_{\leq x}=\{y \in P \mid y \leq x\}$.

Lemma 2.1 (Quillen Fiber Lemma). Let $P$ and $Q$ be posets, and suppose we have an order-preserving map $f: P \rightarrow Q$ such that $\Delta\left(f^{-1}\left(Q_{\leq q}\right)\right)$ is contractible for all $q \in Q$. Then $\Delta(P)$ is homotopy equivalent to $\Delta(Q)$.

Lemma 2.2 (Closure Lemma). Let $P$ be a poset, and suppose that $f: P \rightarrow P$ is a closure operator (i.e. $f(p) \geq p$ and $f^{2}(p)=f(p)$ for all $p \in P$ ). Then $\Delta(P)$ is homotopy equivalent to $\Delta(f(P))$.

If a poset $P$ has unique minimal and maximal elements, denoted by $\hat{0}$ and $\hat{1}$, respectively, then its proper part is $\bar{P}=P \backslash\{\hat{0}, \hat{1}\}$.

The next result can be found e.g. in [4].

Lemma 2.3. Let $L$ be a lattice. Form a simplicial complex $\Sigma$ whose faces are those subsets of the atoms of $L$ whose joins are not the top element. Then $\Delta(\bar{L})$ and $\Sigma$ are homotopy equivalent. 
For simplicial complexes $\Delta_{1}$ and $\Delta_{2}$, let $\Delta_{1} * \Delta_{2}$ denote their join. The following lemma is well-known.

Lemma 2.4. Suppose $\Delta_{i}$ is homotopy equivalent to a wedge of $n_{i}$ spheres of dimension $d_{i}, i=1,2$. Then $\Delta_{1} * \Delta_{2}$ is homotopy equivalent to a wedge of $n_{1} n_{2}$ spheres of dimension $d_{1}+d_{2}+1$.

Finally, we need a convenient collapsibility lemma stated by Björner and Welker [5]. Let $2^{[n]}$ denote the set of subsets of $[n]$. Given $i \in[n]$, define a map $2^{[n]} \rightarrow 2^{[n]}$ by

$$
F \mapsto F \pm i= \begin{cases}F \cup\{i\} & \text { if } i \notin F \\ F \backslash\{i\} & \text { if } i \in F\end{cases}
$$

Lemma 2.5. If $\Delta_{1} \subseteq \Delta_{2}$ are simplicial complexes on the vertex set $[n]$ and there exist vertices $i, j \in[n]$ such that $F \pm i$ maps $\Delta_{2} \backslash \Delta_{1}$ to itself and $F \pm j$ maps $\Delta_{1}$ to itself, then $\Delta_{2}$ is contractible (and so is $\Delta_{1}$ ).

\section{Acyclic subgraphs}

From now on, let $G$ be a fixed directed graph on vertex set $[n]$. Like all graphs in this paper (directed and undirected), $G$ will be assumed to have no loops or multiple edges. Our first object of study is the complex $\Delta_{G}^{A C Y}$ of all acyclic subgraphs of $G$. More precisely, with $E(G)$ denoting the edge set of $G$, we define

$$
\Delta_{G}^{A C Y}=\{F \subseteq E(G) \mid([n], F) \text { has no directed cycle }\} .
$$

Let $\operatorname{Tr}(\cdot)$ denote transitive closure. Define $\operatorname{Pos}_{G}$ to be the following subset of all partial orders on $[n]$ : a poset belongs to $\operatorname{Pos}_{G}$ iff its comparability graph is $\operatorname{Tr}(H)$ for some subgraph $H$ of $G$. Under inclusion, $\operatorname{Pos}_{G}$ is a poset. We denote its unique minimal element, the empty relation, by $\hat{0}$. In the following lemma, the case of $G$ being the complete graph is [5, Lemma 2.1].

Lemma 3.1. The complexes $\Delta\left(\operatorname{Pos}_{G} \backslash\{\hat{0}\}\right)$ and $\Delta_{G}^{A C Y}$ are homotopy equivalent.

Proof. The map $H \mapsto \operatorname{Tr}(H) \cap G$ is a closure operator on $P\left(\Delta_{G}^{A C Y}\right)$. We claim that its image is isomorphic to $\operatorname{Pos}_{G} \backslash\{\hat{0}\}$. To show this, it suffices to check that $\operatorname{Tr}(H)$ can be reconstructed from $\operatorname{Tr}(H) \cap G$; it can, since $\operatorname{Tr}(H)=\operatorname{Tr}(\operatorname{Tr}(H) \cap(G))$. Thus, by Lemma 2.2 , the barycentric subdivision of $\Delta_{G}^{A C Y}$ is homotopy equivalent to $\Delta\left(\operatorname{Pos}_{G} \backslash\{\hat{0}\}\right)$.

Recall that the vertices of any directed graph can be partitioned into strongly connected components: $x$ and $y$ belong to the same component iff there exist directed paths from $x$ to $y$ and from $y$ to $x$. If every vertex belongs to the same component, then the graph is strongly connected.

Björner and Welker stated the following theorem in the case of $G$ being the complete graph only. However, it is straightforward to check that their proof goes through in the more general case, too. 
Theorem 3.2 (See Theorem 2.2 in [5]). If $G$ is strongly connected, then $\Delta\left(\operatorname{Pos}_{G} \backslash\{\hat{0}\}\right)$ is homotopy equivalent to the $(n-2)$-sphere.

It is now straightforward to prove the main result of this section.

Theorem 3.3. If $G$ is a disjoint union of $k$ strongly connected components, then $\Delta_{G}^{A C Y}$ is homotopy equivalent to the $(n-1-k)$-sphere. Otherwise, $\Delta_{G}^{A C Y}$ is contractible.

Proof. If $G$ is not a disjoint union of strongly connected components, then $G$ has an edge $e$ which is not included in any cycle. Thus, $\Delta_{G}^{A C Y}$ is a cone with apex $e$.

Now suppose that $G$ is a disjoint union of $k$ strongly connected components. If $k=1$, then we are done by Theorem 3.2 and Lemma 3.1. Otherwise, $\Delta_{G}^{A C Y}$ is a join of $k$ complexes of this type. Applying Lemma $2.4(k-1)$ times, we conclude that $\Delta_{G}^{A C Y}$ is homotopy equivalent to the sphere of dimension

$$
\sum_{i=1}^{k}\left(a_{i}-2\right)+k-1=n-k-1,
$$

where $a_{i}$ is the number of vertices in the $i$ th component of $G$.

Recall that a quasiorder is a reflexive and transitive relation. The poset (actually a lattice) of quasiorders on $[n]$ is a well-studied object (see e.g. [7]), mainly since quasiorders on $[n]$ correspond in a 1-1 fashion to topologies on $[n]$. The subposet $\operatorname{Pos}_{n}$ of partial orders on $[n]$ then corresponds to the topologies that satisfy the $T_{0}$ separation axiom. Thus, the next corollary can be thought of as a statement about finite topologies.

For a quasiorder $R$ on $[n]$, let $\operatorname{Pos}_{n}^{R}$ be the poset of all posets that are contained in $R$.

Corollary 3.4. Let $R$ be a quasiorder on $[n]$. If $R$ is in fact an equivalence relation with $k$ equivalence classes, then $\Delta\left(\operatorname{Pos}_{n}^{R} \backslash\{\hat{0}\}\right)$ is homotopy equivalent to the $(n-1-k)$-sphere. Otherwise, $\Delta\left(\operatorname{Pos}_{n}^{R} \backslash\{\hat{0}\}\right)$ is contractible.

Proof. There is an obvious correspondence between quasiorders and transitively closed directed graphs. Applying Theorem 3.3 with the graph corresponding to $R$ yields the result via Lemma 3.1.

\section{Not strongly connected subgraphs}

In this section we turn our attention to $\Delta_{G}^{N S C}$, the complex of subgraphs of $G$ that are not strongly connected. More precisely,

$$
\Delta_{G}^{N S C}=\{F \subseteq E(G) \mid([n], F) \text { is not strongly connected }\} .
$$

Again, the case of $G$ being the complete graph was analysed in [5].

Let $\Pi_{G}$ be the subposet of the partition lattice $\Pi_{n}$ consisting of the possible partitions into strongly connected components of subgraphs of $G$. The partition corresponding to 
a graph $H$ is denoted by $\pi(H)$. Clearly, if $\pi(H), \pi\left(H^{\prime}\right) \in \Pi_{G}$, then their join (in $\Pi_{n}$ ) belongs to $\Pi_{G}$. Since $\hat{0} \in \Pi_{G}$, we conclude that $\Pi_{G}$ is a lattice, although it is easy to construct an example showing that $\Pi_{G}$ is not a sublattice of $\Pi_{n}$.

By a minimal cyclic set of $G$, we mean an inclusion-minimal subset $S \subseteq[n]$ with the property that some directed $G$-cycle has $S$ as vertex set. Clearly, such sets correspond to atoms of $\Pi_{G}$. We let $\widehat{G}$ denote the hypergraph on $[n]$ whose edges are precisely the minimal cyclic sets of $G$.

Directed graphs whose minimal cyclic sets all have cardinality two will be important to us. We call such graphs 2-dense. Thus, $G$ is 2-dense iff every cycle contains two vertices that themselves form a cycle in $G$, i.e. iff $\widehat{G}$ is an ordinary graph.

Recall that to any (undirected) graph $H=([n], E)$, one associates the graphical arrangement $\mathcal{A}_{H}$. This is a hyperplane arrangement in $\mathbb{R}^{n}$ containing $|E|$ different hyperplanes, each given by a coordinate equation $x_{i}=x_{j}$ for $\{i, j\} \in E$. Its intersection lattice, $L\left(\mathcal{A}_{H}\right)$, is the lattice of all possible intersections of collections of such hyperplanes, ordered by reverse inclusion.

Theorem 4.1. Suppose that $G$ is 2 -dense. If $\widehat{G}$ is connected, the order complexes $\Delta\left(\overline{\Pi_{G}}\right)$ and $\Delta\left(\overline{L\left(\mathcal{A}_{\widehat{G}}\right)}\right)$ are homotopy equivalent. If $\widehat{G}$ is disconnected, then $\Delta\left(\overline{\Pi_{G}}\right)$ is contractible.

Proof. Suppose that $G$ is 2-dense and denote the edge set of $\widehat{G}$ by $E(\widehat{G})$. Let $\Sigma$ denote the simplicial complex on the vertex set $E(\widehat{G})$ whose simplices are given by the disconnected subgraphs of $\widehat{G}$. By Lemma 2.3 , we have $\Delta\left(\overline{\Pi_{G}}\right) \simeq \Sigma$.

If $\widehat{G}$ is disconnected, then $\Sigma$ is just a simplex, and therefore contractible.

Now suppose that $\widehat{G}$ is connected. Taking transitive closure and then intersecting with $\widehat{G}$ yields a closure operator on $P(\Sigma)$. Its image is isomorphic to the poset of all partitions of $[n]$ that arise as sets of connected components in nonempty disconnected subgraphs of $\widehat{G}$. Clearly, this poset is isomorphic to $\overline{L\left(\mathcal{A}_{\widehat{G}}\right)}$. By Lemma 2.2, the theorem follows.

Remark. Requiring $G$ to be 2-dense is not necessary in the above theorem. If $G$ is not 2-dense, then $\mathcal{A}_{\widehat{G}}$ should be interpreted as the hypergraph subspace arrangement given by $\widehat{G}$. This generalization will not, however, be useful to us later in this paper. For more on hypergraph arrangements and subspace arrangements in general, we refer to the survey [2].

Björner's and Welker's proof of [5, Lemma 3.1] goes through to prove the more general statement below. We state it here to be able to point out where the 2-density assumption is being used. Below, $P \oplus Q$ denotes ordinal sum of posets.

Lemma 4.2 (See Lemma 3.1 in [5]). If $G$ is 2-dense, then $\Delta_{G}^{N S C}$ and $\Delta\left(\operatorname{Pos}_{G} \backslash\{\hat{0}\} \oplus \overline{\Pi_{G}}\right)$ are homotopy equivalent.

Proof. For convenience, let $Q=\operatorname{Pos}_{G} \backslash\{\hat{0}\} \oplus \overline{\Pi_{G}}$. Consider the natural order-preserving surjection $\varphi: P\left(\Delta_{G}^{N S C}\right) \rightarrow Q$ given by

$$
\varphi(H)= \begin{cases}\operatorname{Tr}(H) \in \operatorname{Pos}_{G} \backslash\{\hat{0}\} & \text { if } H \text { is acyclic, } \\ \pi(H) \in \bar{\Pi}_{G} & \text { otherwise. }\end{cases}
$$


In order to use Lemma 2.1, we study the inverse images of $\varphi$.

To begin with, we pick $p \in \operatorname{Pos}_{G} \backslash\{\hat{0}\}$. Clearly, $\varphi^{-1}\left(Q_{\leq p}\right)$ has a unique maximal element, namely the intersection of $G$ and (the comparability graph of) $p$. This element is a cone point, and $\Delta\left(\varphi^{-1}\left(Q_{\leq p}\right)\right)$ is contractible.

Now choose $\tau \in \overline{\Pi_{G}}$. Since $G$ is 2-dense, any non-singleton block of $\tau$ contains a directed $G$-cycle of length two. Without loss of generality, suppose that 1 and 2 form such a cycle. Let $\Delta_{2}=\Delta\left(\varphi^{-1}\left(Q_{<\tau}\right)\right)$ and let $\Delta_{1} \subseteq \Delta_{2}$ be the subcomplex comprising the graphs that contain no directed path from 1 to 2 except possibly the edge $(1,2)$. Now observe that adding the edge $(2,1)$ to $H \in \Delta_{1}$ affects the partition into strongly connected components at worst by merging the part which contains 1 with that which contains 2. This shows that $H \mapsto H \pm(2,1)$ maps $\Delta_{1}$ into itself. Similarly, $H \mapsto H \pm(1,2)$ maps $\Delta_{2} \backslash \Delta_{1}$ into itself. Thus, by Lemma $2.5, \Delta\left(\varphi^{-1}\left(Q_{\leq \tau}\right)\right)$ is contractible, and we are done.

Using $\chi_{\widehat{G}}(t)$ to denote the chromatic polynomial of $\widehat{G}$, we are now in position to state the main theorem. Note that the case of $G$ being not strongly connected is uninteresting since $\Delta_{G}^{N S C}$ is just a simplex in this case.

Theorem 4.3. If $G$ is 2-dense and strongly connected, then $\Delta_{G}^{N S C}$ is homotopy equivalent to a wedge of $(2 n-4)$-dimensional spheres. The number of spheres is $\left|\chi_{\widehat{G}}^{\prime}(0)\right|$.

Proof. By Lemma 4.2 and the definition of ordinal sums, $\Delta_{G}^{N S C} \simeq \Delta\left(\operatorname{Pos}_{G} \backslash\{\hat{0}\}\right) * \Delta\left(\overline{\Pi_{G}}\right)$.

If $\widehat{G}$ is disconnected, $\Delta_{G}^{N S C}$ is contractible by Theorem 4.1. In this case, the linear coefficient of $\chi_{\widehat{G}}(t)$, and thus its absolute value $\left|\chi_{\widehat{G}}^{\prime}(0)\right|$, vanishes as desired. We may therefore assume that $\widehat{G}$ is connected.

It is well-known, see e.g. Rota [13], that the characteristic polynomial of $L\left(\mathcal{A}_{\widehat{G}}\right)$ and the chromatic polynomial of $\widehat{G}$ coincide, i.e.

$$
\chi_{\widehat{G}}(t)=\sum_{x \in L\left(\mathcal{A}_{\widehat{G}}\right)} \mu(\hat{0}, x) t^{\operatorname{dim}(x)},
$$

where $\mu$ is the Möbius function of $L\left(\mathcal{A}_{\widehat{G}}\right)$. Moreover, by a theorem of Björner [3], $\Delta\left(\overline{L\left(\mathcal{A}_{\widehat{G}}\right)}\right)$ has the homotopy type of a wedge of $|\mu(\hat{0}, \hat{1})|$ spheres of dimension codim $(\hat{1})-2$. Since the top element has dimension one in our case, we conclude that $\Delta\left(\overline{L\left(\mathcal{A}_{\widehat{G}}\right)}\right)$, and therefore $\Delta\left(\overline{\Pi_{G}}\right)$, has the homotopy type of a wedge of $(n-3)$-dimensional spheres and that the number of spheres is the absolute value of the linear coefficient of $\chi_{\widehat{G}}(t)$.

Theorem 3.3 shows that $\Delta\left(\operatorname{Pos}_{G} \backslash\{\hat{0}\}\right) \simeq S^{n-2}$, so, by Lemma 2.4, we are done.

Remark. The number of spheres above, i.e. the absolute value of the linear coefficient of the chromatic polynomial of $\widehat{G}$, has a nice interpretation due to Greene and Zaslavsky [9]. It is the number of acyclic orientations of $\widehat{G}$ having a unique fixed sink. See also [8].

Corollary 4.4 (Theorem 1.2 in [5]). The complex of all not strongly connected directed graphs on $[n]$ is homotopy equivalent to a wedge of $(n-1)$ ! spheres of dimension $2 n-4$.

Proof. If $G$ is the complete directed graph, then $\widehat{G}$ is the complete undirected graph. The linear coefficient in its chromatic polynomial is $(-1)^{n-1}(n-1)$ !. 


\section{References}

[1] E. Babson, A. Björner, S. Linusson, J. Shareshian and V. Welker, Complexes of not $i$-connected graphs, Topology 38 (1999), 271-299.

[2] A. Björner, Subspace arrangements, First European Congress of Math., Paris, 1992 (A. Joseph et al., eds.), 321-370, Progr. Math 119, Birkhäuser, Basel, 1994.

[3] A. Björner, Shellable and Cohen-Macaulay partially ordered sets, Trans. Amer. Math. Soc. 260 (1980), 159-183.

[4] A. Björner, Topological methods, in Handbook of Combinatorics (R. Graham, M. Grötschel and L. Lovász, eds.), North-Holland, 1995, 1819-1872.

[5] A. Björner and V. Welker, Complexes of directed graphs, SIAM J. Discrete Math. 12 (1999), 413-424.

[6] X. Dong, Topology of bounded-degree graph complexes, J. Algebra 262 (2003), $287-312$.

[7] M. Erné and J. Reinhold, Intervals in lattices of quasiorders, Order 12 (1995), 375-403.

[8] D. Gebhard and B. Sagan, Sinks in acyclic orientations of graphs, J. Combin. Theory Ser. B 80 (2000), 130-146.

[9] C. Greene and T. Zaslavsky, On the interpretation of Whitney numbers through arrangements of hyperplanes, zonotopes, non-Radon partitions and orientations of graphs, Trans. Amer. Math. Soc. 280 (1983), 97-126.

[10] J. Jonsson, On the topology of simplicial complexes related to 3-connected and Hamiltonian graphs, J. Combin. Theory Ser. A 104 (2003), 169-199.

[11] D. Kozlov, Complexes of directed trees, J. Combin. Theory Ser. A 88 (1999), 112122.

[12] D. Quillen, Homotopy properties of the poset of non-trivial p-subgroups of a group, Adv. Math. 28 (1978), 101-128.

[13] G.-C. Rota, On the foundations of combinatorial theory. I. Theory of Möbius functions, Z. Warsch. Verw. Gebiete 2 (1964) 340-368.

[14] V. Turchin, Homologies of complexes of biconnected graphs, Russian Math. Surveys 52 (1997), 426-427.

[15] M. Wachs, Topology of matching, chessboard and general bounded degree graph complexes, Algebra Universalis, Special issue in memory of Gian-Carlo Rota, to appear. 